$41 \mid 2012$

La mutation du métier de communicant public

\title{
Institution communicante et nouveau management public
}

\section{Élizabeth Gardère}

\section{(2) OpenEdition}

1 Journals

Édition électronique

URL : http://journals.openedition.org/communicationorganisation/3706

DOI : 10.4000/communicationorganisation.3706

ISSN : $1775-3546$

Éditeur

Presses universitaires de Bordeaux

\section{Édition imprimée}

Date de publication : 1 juin 2012

Pagination : 27-39

ISBN : 978-2-86781-821-9

ISSN : $1168-5549$

\section{Référence électronique}

Élizabeth Gardère, «Institution communicante et nouveau management public », Communication et organisation [En ligne], 41 | 2012, mis en ligne le 01 juin 2015, consulté le 04 mai 2019. URL : http:// journals.openedition.org/communicationorganisation/3706; DOI : 10.4000/ communicationorganisation.3706 


\title{
Institution communicante et nouveau management public
}

\author{
Elizabeth Gordère'
}

\section{Institution communicante et nouveau management public}

Depuis la loi de décentralisation (1982), la loi organique relative aux lois de finance (LOLF, 2001), et plus largement la Révision Générale des Politiques Publiques (RGPP, 2007), des réformes touchent l'action publique. L'ambition étatique est de la rendre plus efficace en développant une culture de la performance et de l'évaluation. Le nouveau management public (NMP) est le levier de ce projet gestionnaire dans un contexte de crise politique, financière et économique $(1994,2008)$. Portant sur l'éducation (Université, LRU 2007), la santé (Plan Hôpital 2007, 2012), la sécurité (Police) et bien d'autres domaines, il illustre l'ampleur de la mise en place de ce nouvel esprit de l'action publique. Expression qui fait écho au nouvel esprit du capitalisme (Boltanski : 1999). Certains parlent d'une approche néolibérale de l'État, tandis que d'autres annoncent le Déclin de l'institution (Dubet, 2003).

Un focus en lien avec la réforme des collectivités territoriales (Loi du 16/12/2010) est ici proposé. Après le Royaume-Uni et le Canada, on assiste en France à l'éclatement du modèle traditionnel de la prise de décision publique. L'évolution des objectifs, dispositifs et outils d'accompagnement au changement (Giroux, 2011) génère une reconfiguration de l'institution publique et de ses modes de communication au bénéfice d'une gestion normative, régulée, citoyenne, délibérative, participative. Les « systèmes de représentation politique classiques chargés en principe du bien commun et de l'expertise »(Callon, $2001: 12$ ) sont repensés dans le cadre de la décentralisation à l'échelle d'une gouvernance territoriale locale qui implique des acteurs privés, publics, politiques et citoyens.

1 Elizabeth Gardère est Maître de Conférences Habilité à Diriger des Recherches en Sciences de l'Information et de la Communication. Elle enseigne au Pôle de Gestion des Sciences de Gestion, Université Bordeaux IV. Elle est rattachée au Laboratoire Médiations, Information, Communication, Arts (EA 4426 MICA), Université Bordeaux 3 ; elizabeth.gardere@u-bordeaux4.fr 
Il s'agit d'identifier dans les pratiques, si l'appareil institutionnel peut être géré comme une entreprise avec des objectifs à atteindre, des évaluations, des indicateurs de performance. Cette question remet en cause le postulat distinguant a priori secteur privé et service public. L'axiome est que l'entreprise privée est tenue à une relation de type produits-marché, tandis que l'organisation publique est « contrainte à conserver les mêmes clients et les mêmes produits de base " (David, 2006) tout en assurant une mission de service public. Pourtant, cette convention (Gardère, 2012) s'adapte aux pratiques car « avec l'imprégnation des valeurs du privé dans la sphère publique, le statut d'usager qui marquait la spécificité de la relation entretenue avec le public tend à s'effacer derrière la transposition du rapport plus banal de clientèle " (Chevallier, 1997). Il est à la fois citoyen dans des espaces participatifs et délibératifs (Blondiaux, Sintomer, 2002) et usager-client d'un service (Gardère, 2008).

La méthodologie de recherche est une approche ethnométhodologique (Garfinkel) et emprunte à la théorie de l'action (Parsons). Elle est mise en œuvre depuis dix ans auprès de collectivités territoriales (locales) du Sud-Ouest. Les communes ou municipalités, en tant que divisions administratives, dont l'anonymat est conservé pour des raisons politiques, ont des compétences administratives et prennent en charge les affaires d'intérêt local. Les outils méthodologiques employés sont une immersion dans le terrain, des enquêtes auprès d'instances de pilotage de projets urbains, une collecte d'informations issues de la presse spécialisée (Gazette des communes, Techni Cités, La lettre du cadre territorial), la consultation des procès verbaux de réunions publiques (réunions de concertation, de quartier) et la participation à certains temps forts des projets publics ouverts aux citoyens. D'autres outils d'éthnométhodologie, comme la réflexivité des pratiques et la notion de compétences (Chomsky) rendent compte de cette réalité sociale. La montée en généralité et la conceptualisation permettent de dépasser le constat relatif au nouveau management public (NMP) et aux figures de la communication publique (Gardère, 2009).

\section{Du service public à l'action publique : professionnalisation de l'institution territoriale}

La refonte de l'action publique repose sur les tensions entre une administration de missions (équipement, environnement) qui expérimente le pilotage par projet et une administration de gestion qui s'inscrit dans les prérogatives d'un État souverain. Il s'agit d'une " réorientation autoritaire du mode d'action incitatif de l'État, visant à contrôler les effets de la décentralisation »(Chevalier, 2005 : 224). Suite au bilan de décentralisation de 1982, l'évolution est marquée par le passage du service public à l'action publique avec l'introduction de la logique privée. Celle-ci déplace la mission 
de service public dédiée au contenu vers celle des modalités d'exercice de missions publiques aux temporalités courtes.

Cette orientation envisage l'individu comme un acteur au plus près des affaires et des décisions publiques. La montée en compétences des agents devient un facteur déterminant. En réponse à l'évolution des usagers, les modèles bureaucratiques classiques sont revus : «le problème n'est plus tant de délivrer des biens publics homogènes sur l'ensemble du territoire que de traiter, au cas par cas, des situations complexes et différenciées. La logique bureaucratique avait favorisé les stratégies de clôture des corps professionnels au sein de l'administration » (Le Biannic, 2008 : 348). Les savoirs spécialisés gagnent en polyvalence et le recours à la sous-traitance et aux experts se développe. Cette tendance traduit la volonté d'une "dynamique de rationalisation et de professionnalisation des interventions publiques [...] L'évolution se formalise avec la structuration de filières spécialisées et de nouveaux métiers [...] Elle s'alimente du recrutement d'agents plus qualifiés dans les collectivités locales et de l'invention de formes spécifiques d'expertises localisées » (Pasquier, 2011 : 29). Dans un contexte où les notions de territoire et de proximité sont au cœur de la réorganisation, le transfert des compétences aux collectivités permet d'exercer au plus près des citoyens. Faut-il rappeler qu'avec la LOLF, l'État passe d'une logique de moyens à celle de résultats en réorganisant l'action publique en 34 missions, 133 programmes, 580 actions pour transférer aux collectivités locales plus de compétences et de proximité. Deux axes de subsidiarité sont prioritaires :

- Recomposer les pouvoirs locaux : intercommunalité (compétences de proximité), Région (mutualisation, coopération transnationale Interreg, coopération territoriale);

- Communiquer pour plus d'intelligibilité et d'accessibilité des citoyens.

Par cette requalification, l'État se donne à voir au-delà de ses prérogatives régaliennes. Loin d'afficher l'image d'une organisation politique en délitement à force de réformes et de refontes organisationnelles, l'action publique arbitre entre principes de justice et conventions (Gardère, 2012) pour comprendre les relations d'équivalence entre les parties prenantes (Boltanski, 1991) face aux changements qui touchent l'action publique.

Tandis que le cloisonnement hiérarchique du service public est caractérisé par un respect lent et sinueux des procédures et par l'imbrication des missions de diverses administrations, ce fonctionnement est reconsidéré à l'aune d'un pilotage de projet qui optimise la rapidité des circuits courts de communication et de décision de l'action bureaucratique. Ce point reconsidère les métiers de la communication et la gouvernance (ex. : projet e-administration 2018-2025). À l'occasion de la modernisation des pratiques, proximité, résultat et efficience prévalent. La coordination de l'action publique engage vers de nouvelles instances de management, de contrôle et de régulation (Gardère, 2012) et un double constat s’impose : 
- Relation hiérarchique transformée. Injonction paradoxale du gain d'autonomie mais de l'évaluation des agents. Renforcement des mécanismes de contrôle ;

- Organisation d'entités autour de missions (création d'agences spécialisées, sous-traitances, expertises, partenariats publics/privés), redistribution des responsabilités.

\section{NMP et NCP : figures communicantes d'une institution en réorganisation}

L'expression management public remonte aux années 1970 et relève du modèle entrepreneurial. La littérature française (Aktouf, 1989 ; Bartoli, 1997 ; Thiétart, 1999) considère le NMP comme un ensemble d'actions dans une organisation publique répondant à des méthodes et devant atteindre des objectifs avec une planification et un contrôle.

Le NMP est reconnu comme modèle économique issu des pays anglosaxons ; il inspire la France en termes de communication publique. Il renvoie à la théorie de l'agence dans l'approche des organisations. Fondé sur l'individualisme méthodologique (Boudon), son analyse repose sur l'observation des comportements et stratégies des acteurs du système d'actions. L'une des limites formulée par l'analyse cognitive est de ne pas considérer « la complexité des relations entre l'acteur et le système. Elle ne permet pas de comprendre comment l'acteur est à la fois contraint par le global tout en étant acteur de ce global » (Muller, 2000 : 193). Le collectif n'est pas la somme des intérêts individuels mais une stratégie dans l'intérêt du bien commun assenant des modèles dominants aux acteurs. Dans ce cadre normatif, l'allocation de ressources humaines est dédiée aux « séries d'activités intégrées et interdépendantes " (Aktouf, 1989).

Le NMP « peut servir aux gouvernants à reconfigurer leurs relations avec les gouvernés. Il permet au gouvernement de responsabiliser l'administration, au sens où désormais ce n'est plus lui mais elle que les usagers tiendraient pour responsable de leurs problèmes "(Pollit, in Belorgey, 2010). Levier du changement, le NMP s'appuie sur l'opinion publique, les espaces de délibération et de concertation (Laufer, 1980). Dans cette perspective, le « management public, au sens large, est ce que devient le management lorsque le public prend conscience de l'effet de l'action de l'organisation sur son environnement économique et social. Dès lors, les entreprises privées et publiques peuvent faire du management public. [...] Le management public [...] concerne aussi bien le secteur privé que le secteur public » (Laufer, 1980). Il est une alternative à la lourdeur bureaucratique et à son manque d'innovation. Les citoyens y sont considérés comme des clients à satisfaire : «Ces derniers sont désormais assimilés à des clients (logique consumériste) tandis que les administrateurs deviennent de véritables managers. Cette conception du secteur public - et plus particulièrement de l'administration, reposant sur les $3 \mathrm{E}$ «Economie, Efficacité, Efficience» (Urio, 1998) - est à 
l'opposé de celle de Weber pour lequel la bureaucratie, véritable idéal type, est la condition même de l'efficacité par sa dimension rationaliste. " (Amar, $2007: 3$ ).

Force est de constater la proximité avec le mode projet. Mais ce modèle hybride tient-il compte des particularités de la communication des organisations publiques (Gardère, Lakel, 2009) ? A priori, non. L'un des critères de distinction est la prise en compte, ou pas, de la dimension publique de l'organisation qui se rapprocherait de la notion juridique de droit public qui s'oppose au droit privé. Chevallier (1997) parle du cadre axiologique (intérêt général vs privé) et pratique (droit public vs privé). Considérer, ou non, les organisations publiques comme particulières opère une segmentation :

- Soit l'action publique est une organisation comme une autre (Tassey) où le management public applique les méthodes privées : efficience, rentabilité (Pettigrew, 1997).

- Soit les organisations privées et publiques sont distinctes.

En s'appuyant sur des ressources humaines, la fonction de nouveau communicant public (NCP) est centrale dans le NMP et se décompose en six métiers : « directeurs, chargés de communication ou de publications, graphistes, chefs de projets audiovisuels, multimédias, photographes/vidéastes » (enquête Métiers, Cap'Com, 2011). Le NCP s'inscrit dans « une institutionnalisation et une normalisation qui conduisent à un rattachement de plus en plus fréquent au directeur général des services » (www.cap-com.org/actualité). Le NCP est polymorphe : ingénierie du consentement (politique, animateur, modérateur, coach, médiateur, intégrateur de tendances politiques non plus au niveau du projet politique mais du territoire et des valeurs des projets) ; ingénierie de l'information médiatique, numérique; ingénierie relationnelle (promotion des dispositifs, participation citoyenne, réseaux sociaux) ; ingénierie territoriale (marketing territorial, fonction support aux élus, études d'impact des projets). À cet égard, le NCP s'inscrit dans une communication transversale pilotée par la mesure de l'efficacité des outils, moyens et résultats.

\section{Gouvernance : le cas du NMP territorial}

La notion de gouvernance fait référence à la gestion libérale réduisant le rôle de l'État à ses fonctions de régulation et assurant la répartition des pouvoirs fondée sur une démocratie participative et délibérative (Habermas, 1997) où les individus participent aux affaires locales. Elle a « la capacité qu'ont les sociétés de se doter de systèmes de représentations, d'institutions, de processus et de corps sociaux, considérés comme des outils de contrôle démocratique, de participation aux décisions et responsabilités collectives » (Farinos, 2009: 92) et d'assurer la légitimité face au changement des modalités de prise de décision qui résultent de processus de négociation et de coordination dans une perspective normative et de régulation distincte du modèle traditionnel bureaucratique descendant. Elle donne sens à l'espace public grâce à la 
multiplicité et la diversité des acteurs (Smouts, 1998), la mise en réseaux (Policy networks), la participation, la coordination, la coopération et la concertation.

En exerçant un contrôle sur la société pour gérer le développement local et économique (Bird, 1997), la gouvernance donne forme aux intérêts locaux. Les leviers d'action (Jobert, 2003) sont déclinés à l'échelle territoriale (régulation transversale). En accélérant la réactivité des parties prenantes au niveau des stratégies politiques et de la coopération (Ion, 1990), elle responsabilise les acteurs institutionnels, partenaires privés et citoyens par des accords volontaires qui augurent une gouvernance participative (Froger, 2001), où « la gouvernance est la capacité à produire des décisions cohérentes, à développer des politiques effectives par la coordination entre acteurs publics et non gouvernementaux, dans un univers fragmenté »(Offner, 1999 : 60). Elle est la « somme des différentes façons dont les individus et les institutions, publiques et privées, gèrent leurs affaires communes. C'est un processus continu de coopération et d'accommodements entre des intérêts divers et conflictuels. Elle inclut les institutions officielles et les régimes dotés de pouvoirs exécutoires tout aussi bien que les arrangements informels » (Hufty, 2007 : 29-52).

Trois types de gouvernance se distinguent : d'entreprise, territoriale locale et urbaine. Ce triptyque est le prolongement du néolibéralisme économique à l'œuvre en entreprise. Les points communs sont la transparence, l'auto légitimité des dirigeants, l'avènement d'un esprit démocratique plus normatif, participatif, la responsabilité des citoyens dans la mise en œuvre des projets, la gestion des affaires publiques (Brugvin, 2006) dite bonne gouvernance.

Un focus sur la gouvernance territoriale questionne l'autonomie des processus du développement local destinée à la prise en compte de la société civile dans les affaires locales. L'action publique s'exerce dans un contexte de territoires en recomposition et de redistribution des compétences territoriales des collectivités (subsidiarité, décloisonnement de l'autorité publique). La gouvernance territoriale est un processus d'organisation et de coordination des acteurs valorisant le capital territorial et améliorant la cohésion. Avec la mise en place de centres de décision régulés par des partenariats publics/ privés, « la collectivité locale garde un rôle d'orientation et de pilotage, elle compose avec d'autres institutions, publiques ou privées, obéissant à leurs propres logiques d'intérêt et/ou exerçant des responsabilités sur des domaines de compétences tantôt partagés, tantôt disputés, mais jamais absolument étanches et autonomes » (Bertrand, $2001: 6$ ). Toutefois, « le territoire n'est plus le support exclusif et uniforme des fonctions des gouvernements ; il est désormais remplacé par l'articulation d'espaces multiples plus ou moins spécialisés qui s'équilibrent les uns les autres au lieu de s'inscrire dans une hiérarchie froide et contraignante. » (Badie, 1995 : 220).

La gouvernance territoriale poursuit des actions de décentralisation. L'activité normative s'applique aux dynamiques territoriales dont les processus d'action sont en mutation. Actions de proximité et résultats doivent être en 
adéquation avec la demande citoyenne. Le territoire est un camaïeu d'acteurs privés/publics où la coordination dépasse le cadre formel des politiques publiques et se heurte à l'ordre juridique institutionnel (ex. : la loi de Solidarité et de Renouvellement Urbain - SRU - réforme les règles de l'urbanisme avec l'obligation de quotas de logements sociaux, plans de déplacement et projets de développement urbain qui entrent dans une conception normative et répondent à un objectif de contrôle de l'action).

La régulation politique est définie en sciences politiques comme le droit ou/ et le pouvoir de participation des acteurs et institutions légitimes à la décision pour créer ou appliquer des règles, normes et principes dans un environnement contraint par une histoire. À ce stade, la régulation sociale essuie un échec puisqu'elle limite l'action des pouvoirs publics en termes de normes sociales et développe donc de nouvelles formes de précarité au travail. Une réflexion sur les formes de socialisation et les compétences requises montre que le système bureaucratique n'est plus conforme aux évolutions structurelles et organisationnelles. Le système devient concurrentiel avec l'avènement d'une logique de rationalité managériale. Il est astreint aux enjeux de flexibilité et de mise en concurrence entre des acteurs publics/privés qui investissent la sphère publique le temps d'appels d'offres pour répondre à des projets. Les agents sont soumis à des injonctions paradoxales dans l'exécution de leur tâche ce qui engendre des problèmes de légitimité et compétences qui débouchent sur des mesures d'efficience (évaluation, coût, délai qualité).

La notion d'institution communicante (Bonnet, 2010 : 137-148) démontre la coexistence et l'interdépendance des dimensions organisationnelles, communicationnelles et managériales dont la coopération repose sur les interactions fonctionnelles et symboliques. Dans cette perspective, la prise en compte des socles culturels et formes sociales assure la compréhension des représentations des acteurs de l'organisation grâce à « la construction concertée et l'appropriation des significations liées aux métiers, aux services et aux résultats visés, aux démarches et aux procédures retenues auprès de l'ensemble des acteurs " (Bonnet, 2010 : 144). La coopération s'exprime auprès du citoyen par un mode participatif (Gardère, 2008) qui accélère la temporalité de l'administration dont les délais règlementaires sont peu compatibles avec la temporalité courte citoyenne. Une caractéristique de la gouvernance est la continuité de l'action pour ne pas entrer en tension avec la démocratie représentative dans une situation coopérative des rapports sociaux (Gauchet, 2002). Aussi, loin d'être un processus temporaire, la gouvernance locale est pérenne, structurée et stratégique.

\section{Vers une appropriation du NMP dans les projets urbains}

L'action publique dépend de la coopération lors du glissement vers des logiques privées avec la recomposition de territoires et la redistribution des acteurs : "Dans la conquête par les collectivités territoriales de nouveaux 
pouvoirs de gestion, l'argument démocratique a été rituellement employé : la prise en charge des affaires locales se justifie par la proximité, «opérateur» qui facilite la démocratie. Mais l'emploi de cet argument, s'il pèse dans le jeu de légitimation des élus locaux, ne se limite-t-il pas à des effets de rhétorique ? » (Saez, Bassand : 37).

Les projets d'action publique en zones urbaines et périurbaines sont le foyer d'investigations pour adapter le politique au social dans le respect de normes et règles communes. La collecte de l'information en réunion de quartier est un relais pour mettre en place des systèmes territorialisés de gestion de l'information qui influent sur l'action collective. Il s'agit d'un enjeu de coordination entre les parties prenantes (institution, habitants). Face à ce constat, des interactions systémiques entre les structures sociales, politiques et informationnelles co-construisent des logiques d'acteurs (Fallon, 2009:61), mais c'est la coopération qui apparaît être la solution la plus adéquate à la résolution des projets afin d'éviter trop de médiation.

Les projets urbains sont des instruments d'action publique (Lascoumes, 2004) avec notamment les schémas de cohérence territoriale. La politique de la Ville promue dès 1970 dite développement social des quartiers (DSQ) assure la cohésion sociale. Ses missions variées (projets de rénovation, de bâti, voire de médiation) sont pilotées de manière transversale et citoyenne, et " son mode d'intervention publique valorise la contractualisation et le conventionnement, pour amener différents partenaires publics et privés (État, collectivités, associations, entreprises) à travailler ensemble dans des zones urbaines clairement identifiées " (Vielle Marchiset, 2006 : 1), par le pacte de relance de la ville de 1996 qui confirme le rôle du contrat-ville et insiste sur la nécessité de services marchands de proximité. Il s'agit de mesures incitatives pour faire venir les entreprises dans des zones dédiées. L'ambition affichée est de pallier l'insuffisance de l'intervention publique par la mise en place de missions et de structures parapubliques de proximité. Dans ce cadre, trois axes sont dédiés à la notion de proximité (Colletis, 1999) qui est au cœur des démarches participatives des projets privés/publics :

- Proximité géographique : réflexion sur les notions d'espace, de localisation, de distance comme vecteur de délitement du lien social ou de recomposition de formes de socialisation (Torre, Gilly). La temporalité concerne le temps fragmenté, disloqué, linéaire.

- Proximité organisationnelle : développement des réseaux (TIC, géographique, relationnel), des interactions et de la coopération dans un cadre cognitif à définir (Muller, 2000).

- Proximité institutionnelle : adhésion des acteurs à des règles et représentations communes à l'origine de l'action collective.

Tandis que les municipalités s'inscrivent dans une politique contractuelle avec des dispositifs de financement de type contrat/ville, les services municipaux s'emparent de procédures autres que celles de la bureaucratie 
et s'orientent vers le pilotage de projet. Au résultat opérationnel s'ajoute l'objectif de création d'un lien social local par l'intermédiaire des acteurs des administrations locales (missions locales, maisons de quartier, élus de quartier) pour faire face aux structures plus institutionnelles, tout en mettant l'accent sur les formes participatives porteuses d'intégration. Formes qui relèvent davantage d'une démarche administrative dont découle l'insertion de clauses sociales dans la plupart des marchés publics qui permettent aux collectivités de contrôler l'impact social des actions entreprises sur leur territoire. C'est par exemple le cas du recours à la sous-traitance locale. La participation citoyenne n'étant " qu'un complément local de la démocratie représentative » (Mauger, 2001 : 5-14), il convient d'inclure le volet social dès la phase de démarrage du projet, en garantissant la représentation de l'ensemble des parties prenantes, y compris le citoyen, usager de l'espace public.

Les objectifs de l'action publique conjointe sont collectifs et leur mise en œuvre implique simultanément divers acteurs : État, entreprises, collectivités, associations, syndicats, citoyens. Cette visée opérationnelle suppose davantage de négociation. En effet, l'imbrication des compétences mobilisées dans un espace public est telle qu'il est rare qu'une seule collectivité y soit engagée (Mairie, Communauté urbaine, Conseil Général, Région). C'est par un dialogue en amont et maintenu durant la concertation que le projet se construit malgré les contraintes réciproques des parties prenantes. Par exemple, la réalisation d'un pôle intermodal train-bus implique la Ville, territoire sur lequel se construit le projet, la SNCF, propriétaire des murs et délégataire des transports ferrés, la Région compétente pour les TER, le Conseil Général si des cars interurbains sont prévus, la Communauté Urbaine pour les bus et le tramway. Dans ce cas, la solution optimale décrite par Schelling, ainsi que la stratégie des jeux coopératifs et de négociation de Nash ou encore le point d'équilibre, dit optimum de Pareto, correspondent à une situation où chacun satisfait le plus grand nombre de ses contraintes et de ses attentes dans le respect de l'intérêt collectif tout en dégradant la situation de certaines parties prenantes.

\section{Conclusion}

La gouvernance repose sur une régulation tridimensionnelle culturelle, sociale, économique, complétée d'espaces de négociation et de coopération où la prise de décision s'autonomise. Mais en l'état, la gouvernance n'est pas satisfaisante car elle ne coordonne pas l'ensemble de ces dimensions. La sous-traitance et la coexistence d'acteurs publics/privés dans les projets publics attestent d'une refonte des pratiques institutionnelles où cohabitent les idéaux types de sphères pourtant différentes que sont celles du monde domestique, civique et marchand et de la cité par projet. Les acteurs sont insérés dans des situations conflictuelles et de coordination dans les projets selon le principe de dispute et de mise à l'épreuve (Boltanski, 1991). Dans une 
convention symbolique proche du principe supérieur commun (Boltanski, 1987), les acteurs accordent leur jugement, parfois de manière coercitive et contraignante. C'est le cas du régime contractuel et réglementaire des appels d'offres (relation maître d'ouvrage/maître d'œuvre soumise à ce régime), parfois de façon implicite mais consensuelle lorsque la convention porte sur les conditions du succès du projet en termes de communication, valorisation, reconnaissance et inscription de l'institution publique dans une histoire collective par sa mise en récit. Dans cette dernière hypothèse, les conventions sont un sous-ensemble des règles, contrats et normes visant à coordonner. En tant que dispositifs cognitifs collectifs (Favereau, 1995), elles n'ont pas besoin d'être optimales pour être appliquées.

Finalement, la gouvernance demeure critiquable car l'action publique qui impulse des expériences participatives ne peut pas systématiser les modalités de fonctionnement par des normes. Cela serait contraire à la volonté de l'expression plurielle. Il n'y a donc pas de formes figées des dynamiques participatives au risque de brider la créativité organisationnelle et institutionnelle issue de l'apprentissage collectif. S'il y a bien concertation et dialogue social, les relations de proximité sont discontinues et asynchrones.

Un mode de régulation dans le prolongement de la théorie des conventions est envisageable pour accompagner ce changement organisationnel et donner au métier de communicant public la place qui lui revient (Gardère, 2012). En effet, la fonction de NCP est actuellement occupée par seulement $1 \%$ des agents titulaires ou contractuels de la fonction publique territoriale d'où une réflexion sur le développement de cette fonction, sa transversalité et l'équilibre à trouver dans les missions qui se développent vers le marketing territorial.

\section{BIBLIOGRAPHIE}

AKTOUF O., Le management entre tradition et renouvellement, Paris, Gaëtan Morin, 1989.

BARTOLI A., Le management dans les organisations publiques, Paris, Dunod, 1997.

AMAR A., BERTHIER L., " Le Nouveau Management Public. Avantages et Limites ", Revue RECEMAP, 13, Nice, http://www.unice.fr/recemap/contenurevue/ Articles.html. 2007.

BADIE B., La fin des territoires. Essai sur le désordre international et l'utilité sociale du respect, Paris, Fayard, 1995.

BELORGEY N., L’hôpital sous pression. Enquête sur le Nouveau Management Public, Paris, La Découverte, 2010.

BERTRAND H., GORGEUY., MOQUAY P., «Intégration des formes de proximité dans la gouvernance locale ", Actes des $3^{e}$ journées de la proximité Nouvelles Croissances et Territoires, Paris, 2001. 
BIRD R., VAILLANCOURT F., « Décentralisation financière et pays en développement : concept, mesure et évaluation ", Cahiers de recherche 9714, Université de Montréal, https://papyrus.bib.umontreal.ca/jspui/handle/1866/449. 1997.

BLONDIAUX L., SINTOMER Y., " L'impératif délibératif », Politix, vol. 15, 57, 2002, pp. 17-35.

BOLTANSKI L., CHIAPELLO E., Le nouvel esprit du capitalisme, Paris, Gallimard, 1999.

BOLTANSKI L., THÉVENOT L., Economies de la grandeur, Paris, Gallimard, 1991.

BONNET J., BONNET R., GRAMACCIA G., Management et communication. Mutations, emprunts et résonances, Paris, L'Harmattan, 2010, pp. 137-148.

BRUGVIN TH., «Bonne gouvernance : l'institutionnalisation mondiale de la précarité du travail ", www.organisations.org, $n^{\circ}$ 4, 2006.

CALLON M., LASCOUMES P., BARTHE Y., Agir dans un monde incertain. Essai sur la démocratie représentative, Paris, Seuil, 2001.

Cap'Com, www.médiathèque.cap-com.org/enquête, 2011.

CHEVALLIER J., «La gestion publique à l'heure de la banalisation », Revue Française de Gestion, Paris, octobre 1997, pp. 26-38.

CHEVALLIER G., Sociologie critique de la politique de la ville : une action publique sous influence, Paris, L'Harmattan, 2005.

COLLETIS G., GILLY J.-P., « Construction territoriale et dynamiques économiques », Sciences de la Société, n 48, Paris, 1999, pp. 25-47.

DAVID A., "Quelques enjeux contemporains de la relation de service public ", Revue politique et management public, vol. 24, $\mathrm{n}^{\circ}$ 3, Paris, 2006, pp. 5-12.

DUBET F., Le déclin de l'institution, Paris, Seuil, 2003.

DURAND P., Penser l'action publique, Paris, LGDJ, 1999.

FALLON C., «Entre gestion de crise et vigilance : quand les médias et l'administration publique sont appelés à construire un référentiel partagé ", Communication Eं Organisation, $n^{\circ} 35,2009$, pp. 60-69.

FARINOS D., «Le défi, le besoin et le mythe de la participation à la planification du développement territorial durable : à la recherche d'une gouvernance territoriale efficace ", L’information géographique, vol. 73, Paris, Armand Colin, 2009, pp. 89-111.

FAVEREAU O., "Conventions et régulation ", dans BOYER R., Théorie de la régulation. L'état du savoir, Paris, La Découverte, 1995, pp. 511-520.

FROGER G., «L'aide multicritère à la décision participative : une démarche originale de gouvernance en matière de développement durable ", Eurocongrès "Développement local, développement régional, développement durable : quelles gouvernances? ", Toulouse, 25-26 octobre 2002, p. 1-18.

GARDÈRE E., GARDÈRE J.-P., Démocratie participative et communication territoriale. Vers la micro représentativité, Paris, L'Harmattan, 2008.

GARDÈRE E., LAKEL A. (dir.), « Repenser la communication dans les organisations publiques ", Communication E Organisation, n 35, 2009.

GARDÈRE E., « Normes et conventions : Des dispositifs de régulation aux formes organisationnelles de gouvernance », Colloque international CMN, Université Lille 3, 2012. 
GARDÈRE E., Communication organisationnelle et logiques d'acteurs en mode projet. Du modèle entrepreneurial à la gouvernance publique, HDR, Université Bordeaux 3, 2012.

GAUCHET M., La démocratie contre elle-même, Paris, Gallimard, 2002.

GIROUXN., «Vers une approche processuelle de la communication du changement en organisation ", Actes du colloque Organisation Média, Université Jean Moulin, Lyon, 2004.

HABERMAS J., RAWLS J., Débat sur la justice, Paris, Editions du Cerf, 1997.

HUFTY M. (dir.), Jeux de gouvernance, Genève, IUED, Karthala, 2007.

JOBERT A., DE TERSAC G., La théorie de la régulation sociale de Jean-Daniel Reynaud, Paris, La Découverte, 2003.

KOOIMAN J., Governing as Governance, Londres, Sage, 2003.

LASCOUMES P., LE GALÈS P., (dir.), Gouverner par les instruments, Paris, Presses de Sciences Po, 2004.

LAUFER R., BURLAUD A., Management public, Paris, Dalloz Gestion, 1980.

LE BIANNIC T., VION A., Action publique et légitimités professionnelles, Paris, LGDJ, 2008.

MAUGER G., "Les politiques d'insertion. Une contribution paradoxale à la déstabilisation du marché du travail ", Actes de la recherche en sciences sociales, $n^{\circ} 136$, Paris, 2001, pp. 5-14.

MULLER P., «L'analyse cognitive des politiques publiques : Vers une sociologie politique de l'action publique ", Revue française de sciences politiques, $\mathrm{n}^{\circ}$ 2, 2000, pp. 189-208.

OFFNER J.-M., " La gouvernance urbaine », Réseaux, Institutions et Territoires du LATTS, Paris, ENPC, 1999.

PASQUIER R., Dictionnaire des politiques territoriales, Paris, Presses de Sciences Po, 2011.

PETTIGREW A., " Le NMP conduit à un modèle hybride public-privé ", Revue Française de gestion, Paris, 1997, pp. 113-120.

POLLIT C., The New Public Management in Europe, Palgrave, 2007.

SAEZ G. (dir.), Gouvernance métropolitaine et transfrontalière. Action publique territoriale, Paris, L'Harmattan, 1997.

SMOUTS M.-C., Les nouvelles relations internationales. Pratiques et théories, Paris, Presses de Sciences Po, 1998.

TASSEY G., "Industry and Government strategies for product quality”, International Journal of Technology Management, Vol 4, 2. 1989, pp. 189-203.

THIÉTART R.-A., Le management, Paris, PUF, 1999.

URIO P., "La gestion publique au service du marché », dans HuFTY M. La pensée comptable : État, néolibéralisme, nouvelle gestion publique, Paris, PUF, 1998, pp. 91-124.

VIELLE MARCHISET G., "La politique de la Ville : les enjeux d'une action publique conjointe », www.revue-interrogations.org, 4, 2006, p. 1.

Résumé : Dans le cadre de la Révision Générale des Politiques Publiques (2007), des réformes touchent l'action publique pour la rendre plus efficace en développant une culture de la performance et de l'évaluation. Le nouveau management public (NMP) est 
le levier de ce projet dans un contexte de crise politique, financière et économique (1994, 2008). L'éducation, la santé, la sécurité, parmi d'autres exemples, illustrent l'ampleur de la mise en place de ce nouvel esprit de l'action publique. Afin de gagner en lisibilité, un focus en lien avec la réforme des collectivités territoriales (2010) est ici proposé en termes de gouvernance territoriale et est relatif aux projets urbains. Le passage de la notion de service public à l'action publique implique que l'institution communicante s'empare des dispositifs de proximité et mette en place une politique de communication publique animée par le nouveau communicant public (NCP) au niveau de la vie locale mêlée d'enjeux politiques. Le NMP ne s'envisage pas comme une technique de management, mais comme un enjeu de légitimation de l'action publique face à l'opinion publique, d'où le recours au NCP.

Mots-clés : Action publique, convention, démocratie de proximité, gouvernance territoriale, nouveau management public, nouveau communicant public, projet, régulation.

Abstract : Within the framework of the general revision of the public policies (2007), reforms affect the public action to make them more effective by developing a culture of the performance and the evaluation. New public management (NMP) is the lever of this project in a context of political, financial and economic crisis (1994, 2008). Education, health, security, among other examples, illustrate the scale of the implementation of this new public spirit of the action. A focus on the territorial reform (2010) is proposed here, regarding governance of the urban projects. From the notion of public service to the public action, communication seizes devices of local democracy. The NMP is not a technique of management but a stake in legitimacy of the public action with the support of the NCP.

Keywords : Public action, Convention, Local Democracy, Territorial Governance, New Public management, New Public communicator, Project, Regulation. 
